Investigación original

\title{
DISFUNCIÓN HEPÁTICA EN PREECLAMPSIA. ASOCIACIÓN CON NIVELES ELEVADOS DE GAMAGLUTAMILTRANSFERASA
}

\author{
Jorge Mario Gómez Jiménez, MD*, Jhon Jairo Zuleta Tobón, MD*, \\ Germán Campuzano Maya, MD**, José Cantillo Pabón, MD***
}

Recibido: septiembre 17/2001 - Revisado: noviembre 20/2001 - Aceptado: mayo 30/2002

\section{RESUMEN}

Objetivo: explorar la relación del nivel sérico de gamaglutamiltransferasa con la preeclampsia y sus complicaciones, antes de las 35 semanas de gestación.

Diseño: estudio descriptivo de corte transversal.

Población y lugar: mujeres con menos de 35 semanas de edad gestacional; el grupo I con 55 preeclámpticas y el grupo II con 54 sin preeclampsia, de los hospitales Universitario San Vicente de Paúl y General de Medellín, entre $1^{\circ}$ de septiembre de 1999 y $1^{\circ}$ de septiembre de 2000.

Desenlaces: asociación entre nivel sérico elevado de gamaglutamiltransferasa (GGTe) y preeclampsia (PE).

Análisis estadístico: prueba Kolmogorov-Smirnov para evaluar normalidad, Rho de Spearman para buscar correlaciones entre variables continuas, prueba U de Mann-Witney para comparar medianas, asociación mediante razones de disparidad (RD).

Resultados: no hubo diferencias en las características generales entre los grupos. No se demostró correlación entre el nivel sérico de gamaglutamiltransferasa (GGT) y la edad de la paciente, la edad gestacional, ni con el número de partos previos (Rho de Spearmman 0.03; $0.08 \mathrm{y}$ 0.137 respectivamente). Las medianas de las concentraciones de GGT fueron $22.62 \mathrm{UI} / \mathrm{L}$ (rango 4.76-142.79) para el G I y 17.63 UI/L (rango 7.22-77.86) para el G II, diferencias estadísticamente no significativas ( $\mathrm{p}=0.81$, prueba $\mathrm{U}$ de Mann-Witney). Once (20\%) de las pacientes con PE tuvieron GGTe ( $\geq$ percentil 95 del grupo control, 49.0525 UI/L) y sólo dos controles (3.7\%), $p=0.009$. Se encontró evidencia de asociación entre los niveles iguales o mayores del percentil 95 y preeclampsia, razón de disparidad (RD) 6.5, IC 95\% 1.25 - 45.02.

De 14 pacientes del G I que tuvieron complicaciones atribuibles a la PE, cinco presentaron GGTe, frente a seis de 41 pacientes no complicadas en el mismo grupo ( $p=0.12)$. De 25 madres en el G I que dieron a luz niños con bajo peso para la edad gestacional (BPEG), ocho presentaron GGTe frente a tres con GGTe de 30 madres cuyos hijos nacieron con peso adecuado para la edad gestacional en el mismo grupo, $\mathrm{p}=0.088$.

Conclusión: se definió GGTe para la gestante con un nivel sérico $\geq 49.0525$ UI/L, y se encontró asociación, no causal, con preeclampsia.

Palabras clave: preeclampsia, enzima, Hellp, gamaglutamiltransferasa.

* Ginecoobstetra, docente Universidad de Antioquia. ** Ginecoobstetra, docente Universidad de Antioquia.
*** Hematólogo clínico, Laboratorio Clínico Hematológico. **** Residente II de Ginecoobstetricia, Universidad de Antioquia 


\section{SUMMARY}

Objective: To explore the relationships between elevated serum level of gammaglutamyltransferase (eGGT) and preeclampsia (PE) and related complications before 35 weeks of gestational age.

Methodology: Cross sectional study.

Population and source: 109 pregnant patients before 35 weeks of gestational age [Group I (G I) $n$ = 55 pre-eclamptic patients and group II (G II) $\mathrm{n}=54$ non pre-eclamptic patients] of the hospitals Universitario San Vicente de Paúl and General de Medellín, between September $1^{\text {st }}, 1999$ and September $1^{\text {st }}, 2000$.

Background: Association between eGGT and PE.

Statistical analysis: Kolmogorov-Smirnov's test to evaluate normality, Spearman's Rho to continuous variables; Mann-Witney's U test to compare the medians, Relationships by Odds Ratios.

Results: There were no differences between both groups when analyzing general characteristics. There was no association between eGGT and pregnant age, gestational age nor parity (Spearman's rho $-0.03,0.08$ and 0.137 respectively).

The medians of gammaglutamyltransferase levels (mGGT) were 22.62 UI/L (range 4.76-142.79) and 17.63 (range 7.22 - 77.86) on G I and G II patients, respectively ( $\mathrm{p}=0.81$, Mann-Witney's U test).

Eleven pre-eclamptic pregnant women in G I (20\%) had eGGT [arbitrare cutoff $\geq 49.0525 \mathrm{U} / \mathrm{L}$, mayor or equal than percentile 95 (95p) of the controls] in front to 2 non pre-eclamptic women (3.7\%) in G II ( $\mathrm{p}=0.009$ ), odds ratio (OR) 6.5; 95\% confidence interval (CI) 1.25-45.02.

Fourteen patients in G I had complications attributable to PE, five resulted positive for eGGT in front to 6 positive women of 41 without complications in same group ( $\mathrm{p}=0.12)$.

Of twenty-five intrauterine growth restriction (IUGR) events in G I, eight resulted positive for eGGT compared to three with eGGT of 30 in the same group which did not exhibit IUGR ( $\mathrm{p}=0.088)$. There were six IUGR events in 26 G II negative for eGGT patients that ended its gestation at the source hospitals.

Conclusion: eGGT level in pregnancy was defined $\geq$ p95, like a concentration $\geq 49,0525 \mathrm{UI} / \mathrm{L}$ and evidence of an association, though not causal, with severe PE was found.

Key words: Preeclampsia, gammaglutamyltransferase, Hellp, enzyme.

\section{INTRODUCCIÓN}

El embarazo puede inducir hipertensión arterial en mujeres normotensas o agravar una hipertensión crónica preexistente, produciendo complicaciones maternas y/o fetales. La preeclampsia constituye, junto con las infecciones y las hemorragias, una tríada letal que produce muchas muertes maternas y fetales. Se calcula que en el mundo mueren aproximadamente 50.000 mujeres cada año a causa de eclampsia. Tan alta mortalidad se presenta principalmente en los países subdesarrollados, a causa de una pobre cobertura en los servicios de salud que no permiten a la mayoría de las mujeres acceder a un control prenatal oportuno y adecuado
(1). Aún en países desarrollados como Estados Unidos, la preeclampsia es una amenaza de muerte materna. Según Berg y colaboradores (2), el 18\% de las 1.450 muertes maternas entre 1987 y 1990 en este país se debieron a complicaciones de la preeclampsia. En Colombia, según estadísticas oficiales del Ministerio de Salud (3), la preeclampsia ocupa el primer puesto en mortalidad materna, con una tasa de 42 por 100.000 nacidos vivos.

La preeclampsia presenta compromiso multisistémico en la gestante, generando complicaciones como: insuficiencia renal, coagulopatía, edema agudo de pulmón, 
convulsiones, síndrome de hemólisis-elevación de enzimas hepáticas-trombocitopenia (Hellp); además, el daño de la unidad coriodecidual ocasiona graves complicaciones en el feto: retardo del crecimiento intrauterino (RCIU), sufrimiento fetal agudo (SFA), prematurez y muerte fetal (MF) (4). Por estas razones, esta enfermedad ha sido objeto de múltiples investigaciones con el propósito de esclarecer su etiología y patogénesis y buscar pautas de prevención y tratamiento. Ante la gran cantidad de factores que pueden intervenir en su etiología se ha postulado también su origen multifactorial: alteraciones placentarias y/o enfermedades maternas subyacentes que hayan tenido o no expresión clínica antes del embarazo pueden o no cohalecer para producir la enfermedad (5-7).

En múltiples estudios de pacientes con hepatopatías diversas se ha demostrado la correlación entre niveles altos de fosfatasa alcalina (FA) y de gamaglutamil transferasa (GGT); esta última se considera como el indicador más sensible de enfermedad de vías biliares y como marcador de disfunción hepática por alcohol (8). La GGT es una ectoenzima ligada a las membranas que cataliza la transferencia del grupo gamaglutamil de péptidos como el glutatión a otros aminoácidos, y puede participar en el transporte de aminoácidos. Está compuesta por dos subunidades, ambas codificadas por el mismo ácido ribonucleico mensajero (RNAm). Se encuentra en todo el sistema hepatobiliar y en otros tejidos. Sin embargo los aumentos de esta enzima son inespecíficos y pueden presentarse en afecciones pancreáticas, cardíacas, renales y pulmonares, como la diabetes y el alcoholismo. Su actividad puede incrementarse por sustancias que inducen las enzimas microsomales y se ha propuesto como marcador de alcoholismo. No obstante, su falta de especificidad ha limitado su utilidad clínica (9).

Dadas las alteraciones observadas en las enzimas hepáticas en pacientes con preeclampsia, éstas se utilizan como un parámetro de evaluación de la severidad de la enfermedad. Tradicionalmente se han medido las aminotransferasas y las isoenzimas hepáticas de la deshidrogenasa láctica. En los últimos años se han realizado estudios evaluando el comportamiento de la GGT en la preeclampsia. El objetivo del presente estudio es explorar la posible asociación entre los niveles de gamaglutamiltransferasa y la presencia de preeclampsia.

\section{MATERIALES Y MÉTODOS}

Tipo de estudio: descriptivo de corte transversal.
Población de referencia: gestantes que consultaron en los servicios de urgencias y de alto riesgo obstétrico de los hospitales San Vicente de Paúl y General de Medellín, con preeclampsia moderada o severa (Grupo I) o con gestaciones no complicadas por trastornos hipertensivos de ninguna índole (Grupo II), antes de las 35 semanas de gestación viable.

Muestra: se utilizaron los sueros congelados de pacientes que previamente habían participado en un estudio sobre anticuerpos antifosfolípidos, que generó un banco de sueros $\mathrm{y}$ un archivo con los datos pertinentes (en prensa). Simultáneamente con este estudio se realizó otro sobre la asociación entre hiperhomocisteinemia y preeclampsia.

Muestreo: se seleccionaron 109 pacientes que consultaron en forma consecutiva en las instituciones donde se realizó el estudio; el grupo I estuvo constituido por 55 gestantes con preeclampsia (90.0\% PE severa) y el grupo II por 54 gestantes sin preeclampsia (66\% tenían amenaza de parto prematuro y $44 \%$ ruptura prematura de membranas ovulares).

Criterios de exclusión: gestantes con diagnóstico previo de hipertensión arterial crónica, colagenopatía, nefropatía crónica, embarazo múltiple, embarazo molar, tiroidopatía, epilepsia o antecedente personal de aborto recurrente. También se excluyeron las mujeres que recibían cualquiera de estos medicamentos: clorpromazina, ácido valproico, hidralazina, amoxicilina, propanolol, estreptomicina, procainamida, interferón, quinidina, sulfadoxina, dilantín, pirimetamina.

Criterios de inclusión: se consideró preeclampsia una presión arterial $\geq 140 / 90$ en dos oportunidades separadas por seis horas con proteinuria ( $\geq 300 \mathrm{mg}$ en la orina de 24 horas) y signos y síntomas premonitorios de convulsión y que se encontraron con feto vivo in utero sin malformaciones.

Los criterios para el diagnóstico de Hellp fueron: crenocitos en extendido de sangre periférica o lácticodeshidrogenasa $\geq$ de 1000 U/L, aspartatoaminotransferasa $\quad 0$ alaninoaminotransferasa $\geq 70 \mathrm{U} / \mathrm{L}$, y recuento de plaquetas $\leq$ de $100.000 / \mathrm{ml}$.

El RCIU y el bajo peso para la edad gestacional fueron definidos como peso fetal in utero, estimado por la ultrasonografía de la circunferencia abdominal $o$ al nacer, respectivamente, por debajo del percentil 10 de Hadlock para la edad gestacional definida por amenorrea confiable y/o ultrasonografía en el primer trimestre de la gestación. 
La severidad de la preeclampsia se definió cuando las pacientes tuvieron signos y/o síntomas premonitorios de convulsión, presión arterial diastólica $\geq 110 \mathrm{mmHg}$, oliguria, Hellp y falla renal.

La edad gestacional fue definida por la amenorrea confiable en semanas 0 , en su defecto, por la edad gestacional encontrada por ultrasonografía fetal del primer trimestre de la gestación.

Especificaciones técnicas de la prueba de laboratorio: las muestras se recolectaron dos horas después de un desayuno bajo en grasa, sangrando de vena cefálica 10 cc en tubo seco. Después de separar el coágulo se separó el suero por centrifugación de gravedad y se congeló a $20^{\circ} \mathrm{C}$. Estuvieron congelados por diez meses, después de la primera investigación. El laboratorio realizó confirmación de todos los valores anormales de GGT cuantificada por espectrofotometría cinética utilizando el estuche Gama-GT Cynetic test.Carboxi substrate. Spin React, S.A, Santa Coloma, 7-e-17176 San Esteve de Bas, Gerona, España. Las pruebas fueron realizadas en el Laboratorio Clínico Hematológico, Ltda., Medellín, Colombia.

Consideraciones éticas: este proyecto tuvo la aprobación de los comités de ética de los hospitales participantes. Todas las pacientes firmaron consentimiento para su participación. Además, donaron sus sueros para futuras investigaciones.

Análisis estadístico: se utilizaron los programas SPSS versión 8.0 y Epiinfo 6. Se evaluó la normalidad de la distribución de las variables continuas mediante la prueba de Kolmogorov-Smirnov; como se encontró que no seguían esta distribución, se realizó comparación de los grupos con la prueba $\mathrm{U}$ de Mann-Withney. Para las variables cualitativas se utilizó la prueba chi cuadrado y para los datos en escala ordinal se empleó la prueba chi cuadrado de tendencia.

Resultados: no hubo diferencias entre los grupos en cuanto a las características generales (Tabla 1)

No se halló correlación entre los niveles de GGT con la edad materna (Rho de Spearman 0.03), edad gestacional (Rho 0.08) ni con la paridad (Rho 0.137).

Para las pacientes del G I, la mediana de GGT fue $22.62 \mathrm{UI} / \mathrm{L}$ y los niveles oscilaron entre 4.76 y 142.79. La mediana de GGT en el G II fue $17.63 \mathrm{UI} / \mathrm{L}$ y los niveles oscilaron entre 7.22 y 77.86, sin ningún valor extremo. Estas diferencias no son estadísticamente significativas ( $\mathrm{p}=0.81$, prueba $\mathrm{U}$ de Mann Witney) (Tabla 2 y Fig. 1).

Arbitrariamente se tomó como punto de corte para considerar niveles elevados de gamaglutamiltransferasa (GGTe) el percentil 95 del grupo de pacientes normotensas. Según esta definición, el 20\% (11/55) de las pacientes del G I y sólo el 3.7\% (2/54) del G II presentaron GGTe $(p=0.009)$ (Tabla 2 y Fig. 2). Con este punto de corte, los niveles de GGT $\geq 49.0525$ están asociados con preeclampsia, $\mathrm{RD} 6.5$ (IC $95 \% 1.25$ - 45.02).

Tabla 1

Caracteristicas generales de la población

\begin{tabular}{|ccccc|}
\hline \multicolumn{1}{|c}{ Variable } & Grupo I N = 55 & Grupo $\mathbf{I} \mathbf{n}=\mathbf{5 4}$ & $\mathbf{P}$ \\
\hline Edad ${ }^{(1)}$ & & $26 \pm 7$ & $25 \pm 7$ & $0.48^{(2)}$ \\
Edad gestacional & & $30.4 \pm 2.6$ & $31 \pm 2.7$ & $0.29^{(2)}$ \\
& 1 & $21(38.2)$ & $16(29.6)$ & \\
Grawidez $^{(4)}$ & 2 & $8(14.6)$ & $18(33.3)$ & \\
& 3 & $15(27.2)$ & $8(14.9)$ & \\
& 4 & $11(20.0)$ & $12(22.2)$ & \\
\hline
\end{tabular}

(1) Promedio y desviación estándar

(2) $\mathrm{T}$ student

(9) 02

(4) número $\%$ 


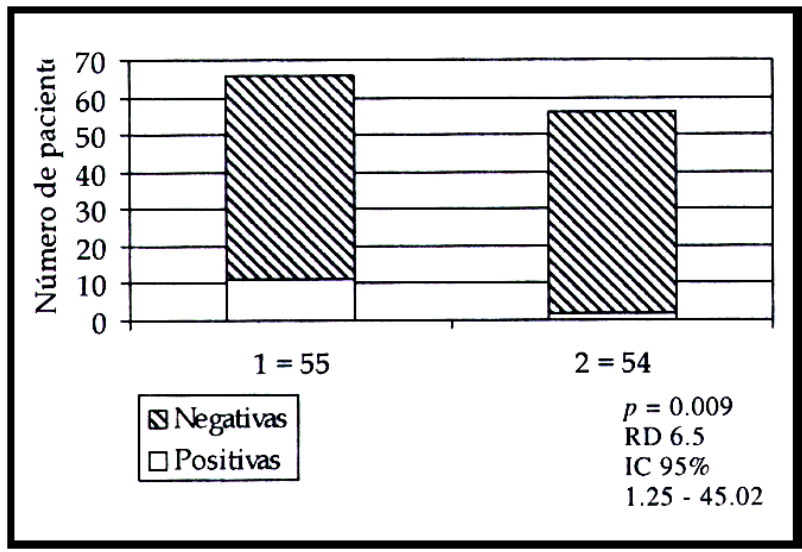

Figura 008i2

Positividad para nivel sérico elevado de gamaglutamil transferasa

Tabla 2

Distribución de niveles de GGT* por percentiles

\begin{tabular}{|ccc|}
\hline Percentiles & Grupo I & Grupo II \\
\hline 5 & 8.8100 & 8.6600 \\
10 & 10.2120 & 9.0700 \\
25 & 13.0500 & 11.1750 \\
50 & 22.6200 & 17.6300 \\
75 & 42.4700 & 30.6475 \\
90 & 64.3940 & 38.2550 \\
95 & 115.7420 & 49.0525 \\
\hline
\end{tabular}

* Gamaglutamiltransferasa en UIL.

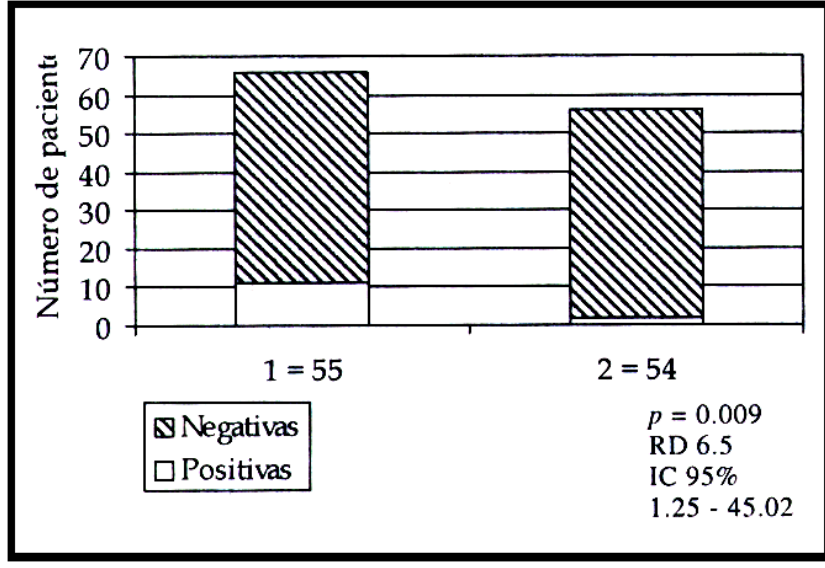

Figura 008i2

Positividad para nivel sérico elevado de gamaglutamil transferasa

Cinco de 14 pacientes en el G I que tuvieron complicaciones atribuibles a preeclampsia, y seis de 41 que no las tuvieron presentaban GGTe, diferencias que no son estadísticamente significativas ( $\mathrm{p}=0.12)$ (Tabla 3$)$.

Ocho de 25 casos de RCIU en el G I tuvieron niveles elevados de GGT frente a 3 de 30 sin RCIU en el mismo grupo. No se demostró asociación ( $\mathrm{p}=$ $0.088)$.

Seis neonatos, hijos de 26 mujeres del G II que terminaron su gestación en los hospitales donde se realizó el estudio, tuvieron RCIU (23.07\%); sus madres fueron negativas para niveles elevados de GGT. Las restantes tuvieron sus hijos en otros centros y no fue posible recuperar los pesos fetales y el resultado materno. De todas maneras fueron dadas de alta en buenas condiciones.

Sólo una de las gestantes negativas para niveles elevados de GGT del grupo II, en quien después se demostró estenosis mitral, se complicó con tromboembolismo pulmonar demostrado por gamagrafía; sobrevivió con anticoagulación.

Discusión: nuestros resultados muestran que una proporción alta de preeclámpticas tienen disfunción hepática (20\%), lo que es sorprendente puesto que sólo tres de las pacientes presentaron síndrome Hellp (una de tres de las pacientes con Hellp tuvo niveles elevados de GGT). La RD de 6.5 para la asociación entre los niveles de GGT por encima del percentil 95 y preeclampsia es alta, yel intervalo de confianza amplio es fiel reflejo del número de pacientes estudiadas. La ausencia de diferencia entre las medianas de GGT entre casos y controles en este estudio puede deberse al tamaño muestral que tuvo bajo poder de detección.

Cabe preguntarse, si un mayor tamaño muestral puede revelar asociación entre GGT y Hellp. A manera de hipótesis, puede pensarse que la gravedad del Hellp depende más de la hemólisis y la trombocitopenia que de la disfunción hepática, o que la terminación de la gestación no permite que tal agravamiento se evidencie por el síndrome Hellp completo. Está por demostrarse, entonces, si los niveles elevados de GGT tienen valor predictivo y diagnóstico para definir el componente disfuncional hepático que hace parte de la preeclampsia y del síndrome Hellp.

$$
\text { Tabla } 3
$$

Complicaciones en gestantes por preeclampsia y niveles séricos de gamaglutamiltransferasa

\begin{tabular}{|ccc|}
\hline Caso & Complicaciones & GGT \\
\hline 1 & Hellp Abrupcio Edema pulmonar & 8.73 \\
2 & Eclampsia & 15.63 \\
3 & Abrupcio* & 50.71 \\
4 & Hellp & 13.36 \\
5 & Sme. Nefrítico & 23.72 \\
6 & Hellp Abrupcio Edema Pulmonar* & 62.81 \\
7 & Abrupcio & 42.47 \\
8 & Muerte fetal & 40.66 \\
9 & Abrupcio & 10.56 \\
10 & Insuf. renal aguda & 15.04 \\
11 & Muerte fetal * & 51.92 \\
12 & Sufrimiento fetal crónico $*$ & 92.19 \\
13 & Sufrimiento fetal agudo $*$ & 49.90 \\
14 & Sufrimiento fetal crónico & 15.93 \\
\hline
\end{tabular}

* Nivel sérico posprandial elevado de gamaglutamiltransferasa (GGTe). 
En relación con la definición de GGTe, debe entenderse que no se han realizado estudios de cohorte que permitan conocer los valores normales esperados para la gestante en los diferentes trimestres de la gestación (tan distintos desde el punto de vista de la volemia y las adaptaciones homeostáticas que conlleva). Por eso en este estudio se definió de acuerdo con un estricto criterio estadístico: por encima del percentil 95 obtenido de embarazadas libres de preeclampsia. De todas maneras llama la atención que la concentración sérica esperada en una mujer fuera del embarazo fluctúa entre 5 y 35 UI/L frente al criterio utilizado en este estudio ( $\geq 49.0525 \mathrm{UI} / \mathrm{L}$ ). Aunque las gestantes control no eran completamente sanas, no hay criterios que hagan pensar que no son representativas de las embarazadas normotensas.

Excepto por incrementos en los niveles de FA, los cambios en la función hepática de las embarazadas sanas no han sido claramente establecidos, porque la mayoría de los estudios no incluyen controles. Al estudiar la función hepática de 103 embarazadas normales, Bacq y cols. (10) encontraron disminución de los niveles de albúmina en todos los trimestres a causa de la hemodilución, y aumento de la FA en el tercer trimestre. La aspartatoaminotransferasa (AST) y los ácidos biliares no difirieron entre embarazadas y no embarazadas. La alaninoaminotransferasa (ALT) fue ligeramente más alta en el segundo trimestre en las embarazadas que en los controles, aunque los valores permanecieron dentro de límites normales; las bilirrubinas tuvieron valores ligeramente bajos en las gestantes en todos los trimestres comparadas con las no embarazadas. Los niveles de GGT fueron más bajos en el segundo y tercer trimestre. El valor normal de GGT en embarazada es de 5-30 $\mathrm{U} / \mathrm{L}$.

Girling y cols. (11) compararon 430 embarazadas normales con 85 pacientes con preeclampsia y encontraron que los niveles séricos de las enzimas hepáticas en embarazadas normales eran menores que los valores de referencia para no embarazadas; la prevalencia de función hepática alterada se presentó en $54 \%$ de las preeclámpticas. Entre las pacientes con preeclampsia, las pruebas de función hepática elevadas se asociaron con proteinuria marcada y bajo recuento de plaquetas.

En un estudio con 202 pacientes, Liu (12) encontró niveles elevados de FA y de GGT en pacientes con preeclampsia severa, mientras que la FA termoestable permaneció en valores bajos. Además, los niveles de FA y leucina aminopeptidasa se incrementaron conforme avanzó la gestación; concluyó que los niveles de FA termoestable pueden reflejar la función placentaria, mientras que la combinación e fosfatasa alcalina y GGT elevadas puede ser útil en la evaluación de la severidad de la preeclampsia y en la predicción del pronóstico materno-fetal.

Es evidente que el carácter ubicuo del órgano blanco de la preeclampsia (riñón, hígado, cerebro, unión coriodecidual) exige del clínico una pesquisa integral que le permita intuir por cuál vía se puede presentar agravamiento. Esto le permitiría tomar medidas tendientes a atenuar la crisis, tales como la expansión de la volemia o la prescripción de fármacos antihipertensivos con acción órganopreferencial (por ejemplo, nimodipino por la circulación cerebral, nifedipina por su acción preferencial bloqueando los canales de calcio dependientes de voltaje en el músculo liso, o labetalol cuando se presenta circulación hiperdinámica). Si antes de que se cumplan criterios para el diagnóstico de Hellp o de necrosis grasa del hígado se puede definir disfunción hepática, tal vez sea posible instaurar medidas preventivas o atenuantes para estos temibles flagelos de la gestante. ¿Cuáles pacientes con preeclampsia tendrán algún grado de disfunción hepática? ¿Cuál es el riesgo de una paciente con disfunción hepática preconcepcional de padecer preeclampsia en la gestación? ¿Cuáles pacientes con preeclampsia y disfunción hepática evolucionarán hacia síndrome Hellp, hacia necrosis grasa o falla hepática? $\mathrm{Ni}$ en su patogénesis ni en sus complicaciones es posible incluir todas las pacientes con preeclampsia dentro de un mismo cuadro clínico. Las respuestas a estas preguntas pueden ayudar en la búsqueda de la prevención $\mathrm{y}$ el tratamiento de algunas de las complicaciones de la preeclampsia.

Puede recomendarse realizar estudios transversales con el propósito de definir el papel de los GGTe en la predicción del agravamiento de la preeclampsia, especialmente por la ruta del síndrome Hellp. De la misma manera puede evaluarse la sensibilidad y especificidad de la GGTe para el diagnóstico de Hellp frente a AST y LDH, y en la predicción del resultado maternofetal.

Agradecimientos: al Laboratorio Clínico Hematológico de Medellín, Colombia, y a la Asociación Antioqueña de Obstetricia y Ginecología, por su generoso y eficiente aporte para la culminación del proyecto.

\section{REFERENCIAS BIBLIOGRÁFICAS}

1. Duley L. Maternal mortality associated with hypertensive disorders of pregnancy in Africa, 
Asia, Latin America and the Caribean. Br J Obstet Gynaecol 1992; 99: 547-52.

2. Berg CJ, Atrash HK, Koonin LM, Tucker M. Pregnancy -related mortality in the United States, 1987-1990. Obstet Gynecol 1996; 88: 161-7.

3. Presidencia de la República, Departamento Nacional de Planeación, Ministerio de Salud y otros. Mortalidad Materna en Colombia. Santafé de Bogotá, agosto 1996.

4. Sibai B, Mencer B, Sarinoglu C. Severe preeclampsia in the second trimester: Recurrence Risk and long-term prognosis. Am Jour Obstet Gynecol 1991; 165: 1408-12.

5. Ness R, Roberts JM. Heterogeneous causes constituting the single syndrome of Preeclampsia: A hypothesis and its implications. Am j Obstet Gynecol 1996; 175: 1365-70.

6. Stone JL, Lockwood CHJ, Berkowitz GS. Risk Factors for Severe Preeclampsia. Obstet and Gynecol 1994; 83: 357-61.

7. Sibai B. Aspectos inmunitarios de la PE. Clin Obstet y Ginecol 1991; 1: 27-33.

8. Churchill D, Kilby MD, Bignell A, et al. Gamma-glutamyl transferase activity in gestational hypertension. Br J Obstet Gynaecol 1994; 101: 251-3.

9. Wetmore LA, Gerard C, Drazen JM. Human lung expresses unique gamma-glutamyl transpeptidase transcripts. Proc Natl Acad Sci USA 1993; 15(90): 7461-5.

10. Bacq Y, Zarka O, Brechot JF y cols. Liver function test in normal pregnancy: a prospective study of 103 pregnant women and 103 matched controls. Hepatology 1996; 23: 1030-4.

11. Girling JC, Dow E, Smith JH. Liver function test in pre-eclampsia: importance of comparison with a reference range derived for normal pregnancy. Br J Obstet Gynaecol 1997; 10: 246-50. 12. Liu J. Clinical significance of serum AKP, HSAP, LAP and gamma-GT determination in highrisk pregnancy. Chung Hua Fu Chan Ko Tsa Chih 1989; 2: 66-9. 